\title{
Short Communication Exercise activity, body size and premenopausal breast cancer survival
}

SM Enger ${ }^{\mathbf{1}, 2}$ and L Bernstein
'D,2
'Department of Research and Evaluation, Kaiser Permanente Medical Care Program, Pasadena, CA 9I I 0 I, USA; ${ }^{2}$ Department of Preventive Medicine,
University of Southern California/Norris Comprehensive Cancer Center, University of Southem California School of Medicine, Los Angeles, CA 90033, USA

We evaluated prediagnosis predictors of breast cancer survival among 717 premenopausal breast cancer patients enrolled in a population-based case-control study and followed for 10.4 years. Using Cox's proportional hazards models, lifetime exercise, weight, and height were not associated with survival. Higher body mass index (trend $P=0.2 \mathrm{I}$ ) and recent exercise activity (trend $P=0.3 \mathrm{I}$ ) were weakly associated with longer survival.

British Journal of Cancer (2004) 90, 2138-2I4I. doi:10.1038/sj.bjc.660I820 www.bjcancer.com

Published online 4 May 2004

(c) 2004 Cancer Research UK

Keywords: physical activity; BMI; weight; breast neoplasms

Evidence suggests that physical activity reduces the risk of breast cancer (IARC, 2002), an effect first reported nearly 20 years ago (Frisch et al, 1985). In addition, high body mass and weight gain increase the risk of postmenopausal breast cancer (International Agency for Research on Cancer, 2002), though a weak inverse relationship between body mass and breast cancer risk has been noted among premenopausal women (Ziegler, 1997; Friedenreich, 2001; Morimoto et al, 2002).

As there is currently interest in whether breast cancer risk factors also impact on survival, we have examined these factors in a population-based cohort of breast cancer patients.

\section{MATERIALS AND METHODS}

Subjects were breast cancer patients who participated in a population-based case-control study among women aged 40 years or younger (Bernstein et al, 1994). Briefly, eligible patients were white or Hispanic women consecutively diagnosed with their first primary in situ or invasive breast cancer from 1 July 1983 through 31 December 1989, identified by the University of Southern California Cancer Surveillance Program (CSP), the populationbased cancer registry for Los Angeles County.

The University of Southern California institutional Review Board (Los Angeles, CA, USA) reviewed and approved the study that complied with all applicable federal regulations governing the protection of human subjects. Study subjects were given a detailed explanation of the study including the risk and benefits. All participants provided signed informed consent to participate in the study.

\footnotetext{
*Correspondence: Dr L Bernstein, USC/Norris Comprehensive Cancer Center 144I Eastlake Avenue, MS 44 (Rm 4449), Los Angeles, CA 90033-0804, USA; E-mail: Ibern@usc.edu.

Received 5 December 2003; revised 3 March 2004; accepted 5 March 2004; published online 4 May 2004
}

In face-to-face interviews, we obtained detailed information about demographics and known or suspected breast cancer risk factors, up to each patient's reference date, defined as 12 months before diagnosis.

We asked patients whether they had ever participated at least twice weekly on a competitive athletic team or in dance or exercise classes, or if they jogged or ran one mile at least twice weekly. For those who responded 'yes', we recorded for each activity the age started and stopped, the type, and the average number of hours per week of participation. We recorded each episode when activities were started and stopped more than once or when the amount of time spent in the activity changed. We computed the number of hours per week each patient participated in all recreational exercise activities beginning with the year of each woman's first menstrual period and ending at the reference date.

Details were collected of height and weight at the reference date and weight at age 18 years. Body mass index (BMI) was computed as weight $(\mathrm{kg})$ divided by the square of height $\left(\mathrm{m}^{2}\right)$ and weight gain as the difference of weight $(\mathrm{kg})$ at the reference date and weight $(\mathrm{kg})$ at age 18 years. Women with zero or negative weight gain were defined as having a zero weight gain.

Details of stage at diagnosis (in situ, localised, regional, distant) and lymph node involvement (number positive and number examined) were abstracted from the records at the CSP and at the hospital of diagnosis.

Follow-up began at interview and ended at the date of death, the date of last follow-up, or 30 June 2000, the end of the study period, whichever was earliest. Vital status and cause of death information was obtained routinely from CSP records, from computerised records of the California Death Certificate Master File and the National Death Index, and by contacting the patients or their relatives by telephone.

Of 969 eligible patients, we interviewed 744 (76.8\%) but then excluded 11 postmenopausal patients, 11 with unknown cancer stage, and five deceased patients with unknown cause of death so that a total of 717 were available for analysis of BMI, weight and height. The first 199 patients enrolled in the case-control study 
did not receive the detailed assessment about lifetime exercise habits leaving 525 patients available for the exercise analyses. Of the 199 patients without detailed lifetime exercise data, 192 were determined to be otherwise eligible and were included in the analyses of body size and breast cancer survival; for these patients, we created a separate exercise activity code.

$\chi^{2} P$-values were used to assess the relationship of patient characteristics at diagnosis with breast cancer survival. Hazard rate ratios (HR) and 95\% confidence intervals $(95 \% \mathrm{Cl})$ were calculated using Cox's proportional hazards methods (Breslow and Day, 1987). Events were deaths due to breast cancer; patients who died from other causes or were alive at the last follow-up were censored at the last follow-up date.

\section{RESULTS}

A total of 454 interviewed patients $(63.3 \%)$ were still alive at the date of last follow-up, with a median follow-up time of 10.4 years (Table 1). We identified 251 breast cancer deaths and 12 deaths due to other known causes among interviewed patients.

Nearly one-half of patients who died of breast cancer were age 35 years or younger at diagnosis compared to just over one-third of those who were alive at the last follow-up or died from other causes (Table 2). As expected, women who died of breast cancer were also more likely to have had an advanced stage and lymph node involvement at diagnosis than other patients. Almost twothirds of patients who died of breast cancer had regional or distant disease at diagnosis compared to less than one-third of other patients; figures were similar for lymph node involvement.

We observed no clear association of lifetime exercise with risk of death from breast cancer, but higher levels of exercise in the year

Table I Years of follow-up of premenopausal breast cancer patients aged 40 years and younger.

\begin{tabular}{|c|c|c|c|}
\hline & \multirow[b]{2}{*}{$\mathbf{N}$} & \multicolumn{2}{|c|}{ Years of follow-up } \\
\hline & & Median & Range \\
\hline All patients & 717 & 10.4 & $0.3-15.1$ \\
\hline Alive at last follow-up & 454 & 11.7 & $0.3-15.1$ \\
\hline Deceased due to breast cancer & 251 & 4.5 & $0.6-14.0$ \\
\hline Deceased due to other causes ${ }^{\mathrm{a}}$ & 12 & 9.0 & $1.0-12.6$ \\
\hline
\end{tabular}

${ }^{a}$ Other causes of death included other cancers $(n=7)$, coronary/cardiovascular disease $(n=2)$, pulmonary disease $(n=2)$, and infections $(n=1)$. before the reference date were weakly associated with a of reduced risk of breast cancer death (Table 3). Compared to those who reported no exercise from their first menses to the reference date, women with the highest levels of activity did not experience a reduced risk of dying from breast cancer. However, compared to women who performed no exercise in the year before the reference date, those who performed at least $5 \mathrm{~h}$ of exercise per week, on average, experienced a $20 \%$ reduction in risk of breast cancer death; this was not statistically significant. We found no marked interaction between risk of death and various combinations of recent activity and lifetime activity levels.

We observed a nearly $25 \%$ reduced risk of death among women in the highest quartile of BMI compared to those in the lowest quartile of BMI, a finding that was not statistically significant (Table 4). Women in the highest quartile of weight experienced a statistically nonsignificant $14 \%$ reduction in risk of dying compared with women in the lowest quartile of weight. Weight gain from age 18 years to the reference date and height were not associated with risk of breast cancer death.

\section{DISCUSSION}

Our findings are consistent with the only other study of physical activity and breast cancer survival which found no relation between risk of breast cancer death and total recreational activity performed in the year before diagnosis (Rohan et al, 1995), similar to our findings for lifetime exercise activity. Our median follow-up time (10.4 years) was nearly twice that of the earlier study (5.5 years), but even with the longer follow-up time we observed no marked association.

As we collected exercise activity data only for those who reported that they had either competed on a sports team, had serious and regular participation in dance, or had run or jogged regularly for at least one mile, some women who were recorded as having not participated in exercise activities may in fact have participated in some activities. The inclusion of these women in the reference category may bias the associations toward the null value, 1.0. However, the intent of the question was to identify the most active women, and activities among women who did not participate in these types of activities are likely to be limited.

Our finding of a weak association of BMI with decreased risk of death is somewhat inconsistent with the body of evidence. Approximately two-thirds of studies have reported an increasing risk of death with increasing BMI or weight, and the remaining studies generally reported no association (Goodwin and Boyd, 1990; Rock and Demark-Wahnefried, 2002). The women in the

Table 2 Characteristics of eligible breast cancer patients aged 40 years or younger at diagnosis.

\begin{tabular}{|c|c|c|c|c|c|}
\hline \multirow[b]{2}{*}{ Characteristics at breast cancer diagnosis } & \multicolumn{2}{|c|}{ Still living or died from other known causes $(n=466)$} & \multicolumn{2}{|c|}{ Died of breast cancer $(n=25 I)$} & \multirow[b]{2}{*}{$\chi^{2} P$} \\
\hline & $\mathbf{n}$ & $\%$ & $\mathrm{~N}$ & $\%$ & \\
\hline $21-25$ & 6 & 1.3 & 11 & 4.4 & \\
\hline $26-30$ & 31 & 6.7 & 23 & 9.2 & \\
\hline $31-35$ & 132 & 28.3 & 85 & 33.9 & \\
\hline $36-40$ & 297 & 63.7 & 132 & 52.5 & 0.005 \\
\hline Localised & 255 & 54.7 & 85 & 33.9 & \\
\hline Regional & 139 & 29.8 & $|4|$ & 56.2 & \\
\hline Distant & 4 & 0.9 & 22 & 8.7 & $<0.0001$ \\
\hline \multicolumn{6}{|l|}{ Axillary lymph node involvement } \\
\hline Yes & 139 & 29.8 & 156 & 62.1 & \\
\hline No & 323 & 69.3 & 88 & 35.1 & $<0.0001$ \\
\hline Unknown & 4 & 0.9 & 7 & 2.8 & \\
\hline
\end{tabular}


Table 3 Risk of dying from breast cancer in relation to physical activity from first menses to reference date ${ }^{\mathrm{a}}$ and during year before the reference date, among breast cancer patients aged 40 years or younger at diagnosis

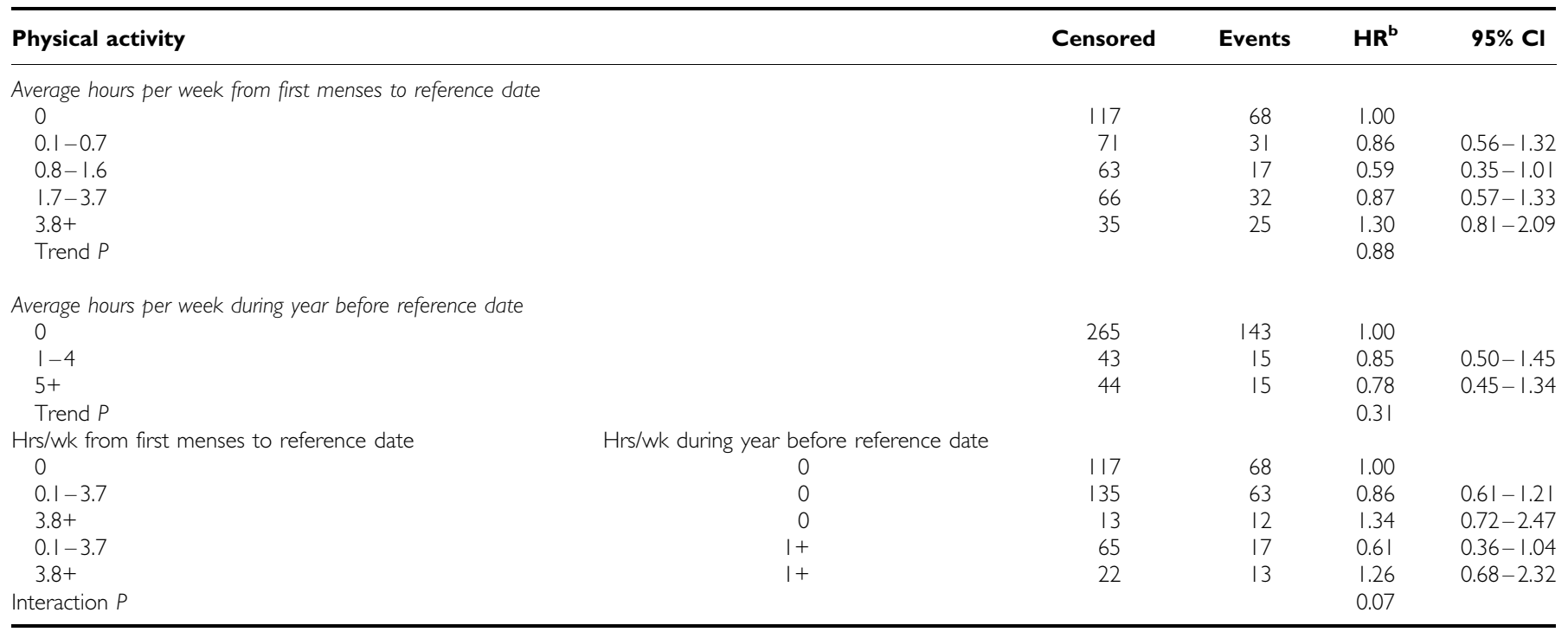

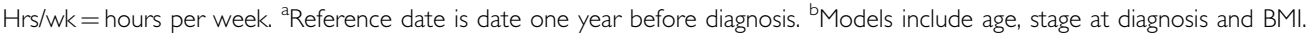

Table 4 Body size indicators ${ }^{\mathrm{a}}$ and risk of breast cancer death among breast cancer patients aged 40 years or younger at diagnosis

\begin{tabular}{|c|c|c|c|c|}
\hline & Censored & Events & $H_{R^{b}}$ & $95 \% \mathrm{Cl}$ \\
\hline \multicolumn{5}{|l|}{ BMI $\left(\mathrm{kg} \mathrm{m}^{-2}\right)$} \\
\hline$<20.4$ & 110 & 65 & 1.00 & \\
\hline $20.4-21.9$ & 117 & 64 & 0.79 & $0.56-1.12$ \\
\hline $22.0-24.8$ & 113 & 66 & 0.89 & $0.63-1.26$ \\
\hline $24.9+$ & 118 & 64 & 0.76 & $0.53-1.07$ \\
\hline Trend $P$ & & & 0.21 & \\
\hline \multicolumn{5}{|l|}{ Weight (kg) } \\
\hline$<54.1$ & 116 & 57 & 1.00 & \\
\hline $54.1-60.9$ & 115 & 81 & 1.10 & $0.78-1.54$ \\
\hline $61.0-68.1$ & 110 & 54 & 0.87 & $0.60-1.26$ \\
\hline $68.2+$ & 125 & 59 & 0.86 & $0.60-1.23$ \\
\hline Trend $P$ & & & 0.21 & \\
\hline \multicolumn{5}{|c|}{ Weight gain (kg) } \\
\hline 0 & 73 & 33 & 1.00 & \\
\hline $0.1-5.0$ & 181 & 112 & 0.97 & $0.66-1.43$ \\
\hline $5.1-10.0$ & 104 & 49 & 0.81 & $0.52-1.24$ \\
\hline $10+$ & 108 & 57 & 0.93 & $0.61-1.42$ \\
\hline Trend $P$ & & & 0.53 & \\
\hline \multicolumn{5}{|l|}{ Height (m) } \\
\hline$<1.60$ & 100 & 57 & 1.00 & \\
\hline $1.60-1.64$ & 133 & 68 & 0.92 & $0.65-1.30$ \\
\hline $1.65-1.68$ & 124 & 59 & 0.84 & $0.58-1.21$ \\
\hline $1.69+$ & 109 & 67 & 1.17 & $0.81-1.69$ \\
\hline Trend $P$ & & & 0.52 & \\
\hline
\end{tabular}

aody size indicators at reference date ( 1 year before diagnosis), except for weight gain which was increase in weight from age 18 years to the reference age. 'bodels include age, stage at diagnosis and physical activity (combination of average hours per week during the reproductive years and average hours per week during the year before the reference date); in addition, models of weight and height are mutually adjusted.

current study are among the youngest reported in the literature and as a group had a very low median BMI prior to diagnosis. It is possible that other factors, such as oestrogen receptor status, account for our findings, which are also consistent with no association.
A concern in this as in any follow-up study is loss to follow-up. The patients who were lost to follow-up early on may have differed from those with complete follow-up, leading to bias in the results. However, we were able to follow-up $79 \%$ of the patients to the end of the follow-up period and $92 \%$ to within 2 years of this point, so it is unlikely that such losses resulted in substantial biases. Another possible limitation is that recall of past exercise activities resulted in some misclassification, particularly those decades before interviews. However, we obtained activity information only from those who indicated that they had participated in serious sports activities or dancing, which allowed us to identify women engaged in strenuous exercise. Although some misclassification undoubtedly occurred, it is likely that our methods resulted in separation of the most active from the least active cohort members. Breast cancer diagnosis may have influenced recall of past exercise activities, but since all patients in the cohort were interviewed after learning their diagnoses, they should all have been similarly affected. All women in the cohort were interviewed prior to the widespread publication of papers indicating that exercise activity was associated with reduced breast cancer risk.

It is unclear whether prediagnosis physical activity levels are markers of postdiagnosis activity levels or whether postdiagnosis physical activity influences survival. A recent study reported $24-50 \%$ decreases in postdiagnosis compared to prediagnosis physical activity levels of breast cancer patients (Irwin et al, 2003), but postdiagnosis levels were assessed within 1 year of diagnosis. We interviewed 374 of the surviving breast cancer patients from this study in 1998-1999 as part of a follow-up study and found that, on average, surviving women had increased their activity levels in the years following their diagnoses.

Overall, we observe no association of lifetime exercise with breast cancer survival and only a weak association of higher levels of recent exercise with breast cancer survival. In contrast, we find a weak association of higher BMI levels with reduced risk of breast cancer death. It remains unclear whether recent, prediagnosis activity, and body size levels are indicators of postdiagnosis levels. Further examination of physical activity and body size during both pre- and postdiagnosis periods will improve our evaluation of the impact of these factors on breast cancer survival. 


\section{ACKNOWLEDGEMENTS}

We are grateful to all the women who participated in this study and to the physicians who allowed us to contact their patients. We also thank Rosemarie Hanisch for data collection, and Jane Sullivan-Halley for computing and statistical assistance. This study was supported by Public Health Service Grants CA44546 and CA17054 and by the SEER Contract N01-PC-67010 from the National Cancer institute, National

\section{REFERENCES}

Bernstein L, Henderson BE, Hanisch R, Sullivan-Halley J, Ross RK (1994) Physical exercise and reduced risk of breast cancer in young women. $J$ Natl Cancer Inst 86: 1403-1408

Breslow NE, Day NE (1987) Statistical Methods in Cancer Research. Volume II-The Design and Analysis of Cohort Studies. IARC Scientific Publication No. 82. Lyon, France: International Agency for Research on Cancer

Friedenreich CM (2001) Review of anthropometric factors and breast cancer risk. Eur J Cancer Prev 10: 15-32

Frisch RE, Wyshak G, Albright NL, Albright TE, Schiff I, Jones KP, Witschi J, Shiang E, Koff E, Marguglio M (1985) Lower prevalence of breast cancer and cancers of the reproductive system among former college athletes compared to non-athletes. Br J Cancer 52: 885-891

Goodwin PH, Boyd NF (1990) Body size and breast cancer prognosis: a critical review of the evidence. Breast Cancer Res Treat 16: 205-214

International Agency for Research on Cancer (2002) Weight Control and Physical Activity. IARC Handbooks of Cancer Prevention, Vol 6. Lyon, France: IARC Press
Institutes of Health, Department of Health and Human Services; and by the California Public Health Foundation, Subcontract 050-F-8709, which is supported by the California Department of Health Services as part of its statewide cancer-reporting program mandated by Health and Safety Code Sections 210, 211.3. 210 and 211.3. Dr Enger was supported by funds from the California Breast Cancer Research Program of the University of California, Grants 1FB-0341 and 3FB-0097.

Irwin ML, Crumley $\mathrm{D}$, McTiernan $\mathrm{A}$, Bernstein $\mathrm{L}$, Baumgartner $\mathrm{R}$, Gilliland FD, Kriska A, Ballard-Barbash R (2003) Physical activity levels before and after a diagnosis of breast carcinoma: the Health, Eating, Activity, and Lifestyle (HEAL) study. Cancer 97: $1746-1757$

Morimoto LM, White E, Chen Z, Chlebowski RT, Hays J, Kuller L, Lopez AM, Manson J, Margolis KL, Muti PC, Stefanick ML, McTiernan A (2002) Obesity, body size, and risk of postmenopausal breast cancer: the Women's Health Initiative (United States). Cancer Causes Control 13: $741-751$

Rock CL, Demark-Wahnefried W (2002) Nutrition and survival after the diagnosis of breast cancer: a review of the evidence. J Clin Oncol 20: $3302-3316$

Rohan TE, Fu W, Hiller JE (1995) Physical activity and survival from breast cancer. Eur J Cancer Prev 4: 419-424

Ziegler RG (1997) Anthropometry and breast cancer. J Nutr 127: 924S - $928 \mathrm{~S}$ 